\title{
Electrical properties of the system lanthanum lead cobalt titanium oxide
}

\author{
DEVENDRA KUMAR $\dagger$, Ch. DURGA PRASAD and \\ OM PARKASH*
}

School of Materials Science and Technology, †Department of Ceramic Engineering, Institute of Technology, Banaras Hindu University, Varanasi 221005 , India

MS received 1 April 1987

\begin{abstract}
Measurements of DC electrical resistivity and Seebeck coefficient on the perovskite system $\mathrm{La}_{1-x} \mathrm{~Pb}_{x-\delta} \mathrm{Co}_{1-x} \mathrm{Ti}_{x} \mathrm{O}_{3-\delta}$ for $0.2 \leqslant x \leqslant 0.9$ have been made in the temperature range $300-800 \mathrm{~K}$. AC conductivity, $\sigma_{\mathrm{a} . \mathrm{c}}$, of all the samples were measured as a function of temperature $(300-573 \mathrm{~K})$ and frequency $(1 \mathrm{kHz}-1 \mathrm{MHz})$. DC resistivity behaviour of all the samples is similar. However, the resistivity value, which varies over 5-6 orders of magnitude, depends on both the composition $x$ and the structure of the samples. All the samples exhibit $p$-type electronic conduction. The value of Seebeck coefficient, $\alpha$, for samples with $x \leqslant 0.5$ initially increases with temperature up to a particular temperature. Above this temperature, the behaviour of $\alpha$ for samples with $x \leqslant 0.5$ and for samples with $x>0.5$ over the entire temperature range is similar to that of $\mathrm{La} \mathrm{CoO}_{3}$. All the samples exhibit frequency-dependent a.c. conductivity at low temperatures.
\end{abstract}

Keywords. Perovskite; resistivity; Seebeck coefficient; hopping; lanthanum lead cobalt titanium oxide

\section{Introduction}

Lanthanum cobaltate, $\mathrm{LaCoO}_{3}$, exhibits interesting electrical and magnetic properties due to co-existence of low-spin and high-spin cobalt ions in it (Raccah and Goodenough 1967; Bhide et al 1972). Cobalt ions which exist in the diamagnetic lowspin configuration $t_{2 g}^{6} e_{g}^{0}$ at very low temperatures transform to paramagnetic highspin state $t_{2 g}^{4} e_{q}^{2}$ upto $200 \mathrm{~K}$. Above this temperature, electron transfer occurs from the high-spin $\mathrm{Co}^{3+}$ ions to low-spin $\mathrm{Co}^{\mathrm{III}}$ ions to give intermediate charge transfer states like $\mathrm{Co}^{2+}, \mathrm{Co}^{\mathrm{IV}}$ etc. Around $1200 \mathrm{~K}, \mathrm{LaCoO}_{3}$ undergoes a first order transition from localized to collective behaviour of $d$-electrons.

Replacement of $\mathrm{La}^{3+}$ in $\mathrm{LaCoO}_{3}$ by $\mathrm{Sr}^{2+}$ changes its properties remarkably (Bhide et al 1975). Thus the system $\mathrm{La}_{1-x} \mathrm{Sr}_{x} \mathrm{CoO}_{3}$ becomes ferromagnetic at low temperatures for $x>0.125$ due to $\mathrm{Co}^{4+}-\mathrm{O}-\mathrm{Co}^{3+}$ interactions; $\mathrm{Co}^{4+}$ ions being generated by $\mathrm{Sr}^{2+}$ substitution to maintain electrical neutrality. However, if the electrical neutrality is maintained by the simultaneous substitution of $\mathrm{Ti}^{4+}$ for $\mathrm{Co}^{3+}$ in the above system, no ferromagnetism is observed as indicated by the studies of electron-transport properties and Mössbauer spectra of the system $\mathrm{La}_{1-x} \mathrm{Sr}_{x}$ $\mathrm{Co}_{1-x} \mathrm{Ti}_{x} \mathrm{O}_{3}(0-0 \leqslant x \leqslant 0 \cdot 5)$ (Bahadur and Om Parkash 1983; Kumar et al 1985). This is due to the absence of $\mathrm{Co}^{4+}-\mathrm{O}-\mathrm{Co}^{3+}$ links in this system. Further, the localized character of $d$-electrons increases with increasing $x$. On the other hand, substitution of $\mathrm{Ti}^{4+}$ for $\mathrm{Co}^{3+}$ in $\mathrm{LaCoO}_{3}$ gives rise to high-spin $\mathrm{Co}^{2+}$ ions in order to maintain a charge neutrality in the system $\mathrm{LaCo}_{1-x} \mathrm{Ti}_{x} \mathrm{O}_{3}(0<x \leqslant 0 \cdot 5)$.

Recently formation of solid solution $\mathrm{La}_{1-x} \mathrm{~Pb}_{x} \mathrm{Co}_{1-x} \mathrm{Ti}_{x} \mathrm{O}_{3}$ has been reported for

*To whom all correspondence should be addressed. 
all values of $x$ (Om Parkash et al 1987). These samples had some lead deficiency in them. In this paper we report the detailed electrical properties of this system in the composition range $0 \cdot 20 \leqslant x \leqslant 0 \cdot 90$.

\section{Experimental}

All the samples were prepared by ceramic method starting with $A R$ grade lanthanum oxalate, lead monoxide, cobalt oxalate and titanium dioxide. The method of preparation has been reported earlier (Om Parkash et al 1987). Formation of a single phase in all the samples was established by $\mathrm{x}$-ray diffraction. Chemical analysis was carried out using an atomic absorption spectrophotometer (Perkin-Elmer). Total concentration of cobalt was also determined gravimetrically using the mercury thiocyanate method. Both the results were identical. DC resistivity and Seebeck coefficient was measured as a function of temperature on sintered pellets pressed between spring-loaded platinum foils employing a digital electrometer (Keithley 616). AC conductivity of sintered pellets coated with silver paint on both sides was measured using an impedance analyzer (HP 4192 LF) as a function of temperature and frequency.

\section{Results and discussion}

All the samples in the study were single phase materials. Analysis of $x$-ray diffraction data showed that the overall symmetry was rhombohedral for $x \leqslant 0.30$; cubic for 0.30 $<x \leqslant 0.80$ and tetragonal for $x \geqslant 0.90$. In an earlier report, composition with $x=0.30$ has been reported to have a cubic symmetry (Om Parkash et al 1987). A more careful analysis shows that it has a slight rhombohedral distortion with $a=5.525 \AA$ and $\alpha=60^{\circ} .10^{\prime}$. Similarly compositions with $0.5 \leqslant x \leqslant 0.8$, have an overall cubic symmetry. However, the asymmetry of diffraction lines shows that these samples have a slight tetragonal distortion with $c / a$ ratio very close to one. This is further supported by the observed anomalies in their behaviour of dielectric constant as a function of temperature (Om Parkash et al 1987). Similar results have also been reported in the case of lanthanum-substituted lead zirconate titanate (Wolters and Burggraaf 1974).

Chemical analysis indicates that all the samples were lead-deficient. This is due to the loss of $\mathrm{PbO}$ during calcination and sintering. Once prepared, it was found that these samples are thermally stable at high temperatures $(\sim 1100 \mathrm{~K})$ where no detectable loss in weight was observed even after heating for several hours.

Effect of substitution of $\mathrm{Ti}^{4+}$ and $\mathrm{Pb}^{2+}$ on the valence state of cobalt ions in $\mathrm{LaCoO}_{3}$ can be viewed as follows: alone substitution of $\mathrm{Ti}^{4+}$ promotes the generation of divalent cobalt ions, $\mathrm{Co}^{2+}$, as mentioned in $\S 1$. However, the simultaneous substitution of $\mathrm{Pb}^{2+}$ (on $\mathrm{La}^{3+}$ site) along with $\mathrm{Ti}^{4+}$ (on $\mathrm{Co}^{3+}$ site) reverses this process by forcing the cobalt ions to remain in the trivalent state. Due to loss of $\mathrm{PbO}$ during preparation, the proportionate amount of $\mathrm{Pb}^{2+}$ is less than $\mathrm{Ti}^{4+}$ as represented in the formula $\mathrm{La}_{1-x} \mathrm{~Pb}_{x} \mathrm{Ti}_{x} \mathrm{O}_{3}$ in all the samples. Therefore, the reversal of $\mathrm{Co}^{2+}$ to $\mathrm{Co}^{3+}$ as mentioned above may not be complete. It implies that some cobalt ions are in the divalent state similar to that in the system $\mathrm{LaCo}_{1-x} \mathrm{Ti}_{x} \mathrm{O}_{3}$ at least upto $x \leqslant 0 \cdot 50$. Exact determination of relative concentrations 
of $\mathrm{Co}^{2+}$ and $\mathrm{Co}^{3+}$ ions was not possible by iodimetry due to the complex nature of the system. We can write the chemical formula of our samples as $\mathrm{La}_{1-x} \mathrm{~Pb}_{x-\delta} \mathrm{Co}_{1-x-y}^{3+} \mathrm{Co}_{y}^{2+} \mathrm{Ti}_{x}^{4+} \mathrm{O}_{3-\delta-y / 2}$. Values of $\delta$ for all the samples are given in table 1. It is interesting to note that the $\delta$ value is 0.21 for samples with $x>0.50$. The density of all the samples determined by water displacement method is given in table 1.

Typical plots of Seebeck coefficient, $\alpha$, as a function of temperature, $T$, are shown in figure 1. All the samples were found to have positive values of $\alpha$ over the entire

Table 1. Lead deficiency $\delta$, density and activation energy $E_{a}$ for conduction for various samples in the system $\mathrm{La}_{1-x} \mathrm{~Pb}_{x-\delta} \mathrm{Co}_{1-x} \mathrm{Ti}_{x} \mathrm{O}_{3-\delta}(0-2 \leqslant x \leqslant 0 \cdot 9)$

\begin{tabular}{cccc}
\hline$x$ & $\delta$ & $\begin{array}{c}\text { Density } \\
(g / \mathrm{cc})\end{array}$ & $E_{a}(\mathrm{eV})$ \\
\hline 0.20 & 0.16 & 6.18 & 0.30 \\
0.30 & 0.20 & 6.23 & 0.48 \\
0.40 & 0.23 & 6.28 & 0.46 \\
0.50 & 0.25 & 6.59 & 0.41 \\
0.60 & 0.20 & 6.92 & 0.47 \\
0.70 & 0.21 & 6.97 & 0.58 \\
0.80 & 0.21 & 7.16 & 0.65 \\
0.90 & 0.21 & 7.20 & 0.55 \\
\hline
\end{tabular}

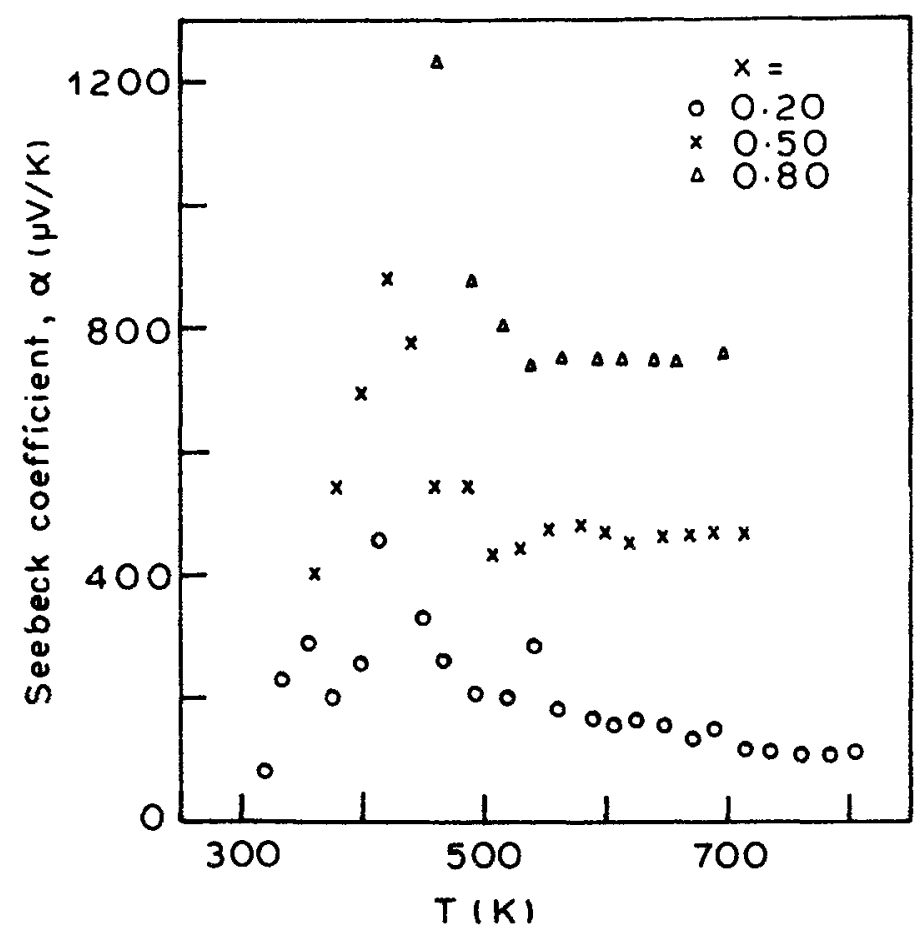

Figure 1. Variation of Seebeck coefficient $\alpha$ with temperature for samples with $x=0.20,0.50$ and 0.80 in the system $\mathrm{La}_{1-x} \mathrm{~Pb}_{x-8} \mathrm{Co}_{1-x} \mathrm{Ti}_{x} \mathrm{O}_{3-\delta}$. 
range of temperature indicating that the holes are the majority charge carriers. For $0.20 \leqslant x \leqslant 0.50, \alpha$ initially increases with the increasing temperature and peaks at a particular temperature $T_{\alpha}$. Above $T_{\alpha}, \alpha$ decreases and finally becomes constant with respect to temperature. For $x>0.50, \alpha$ initially decreases rapidly with temperature and finally becomes constant with respect to temperature similar to samples with $x \leqslant 0.50$. Measurements of $\alpha$ below $400 \mathrm{~K}$ were not possible for $x>0.50$ because of their high resistivity. The value of $\alpha$ increases with $x$. Variation of $\log \rho_{\mathrm{d} . c}$ with $1000 / T$ for all the samples is shown in figure 2 . The resistance of the samples was found to be independent of time at a steady temperature in the temperature range of measurements. This indicates that the electrical conductivity is mainly electronic in nature. Two regions are observed in $\log \rho_{\mathrm{d} . \mathrm{c}} \mathrm{vs} 1000 / T$ plots of all the samples. The resistivity in region 1 decreases sharply with temperature in accordance with the Arrhenius relationship:

$$
\rho=\rho_{01} \exp \left(-E_{a} /(k T),\right.
$$

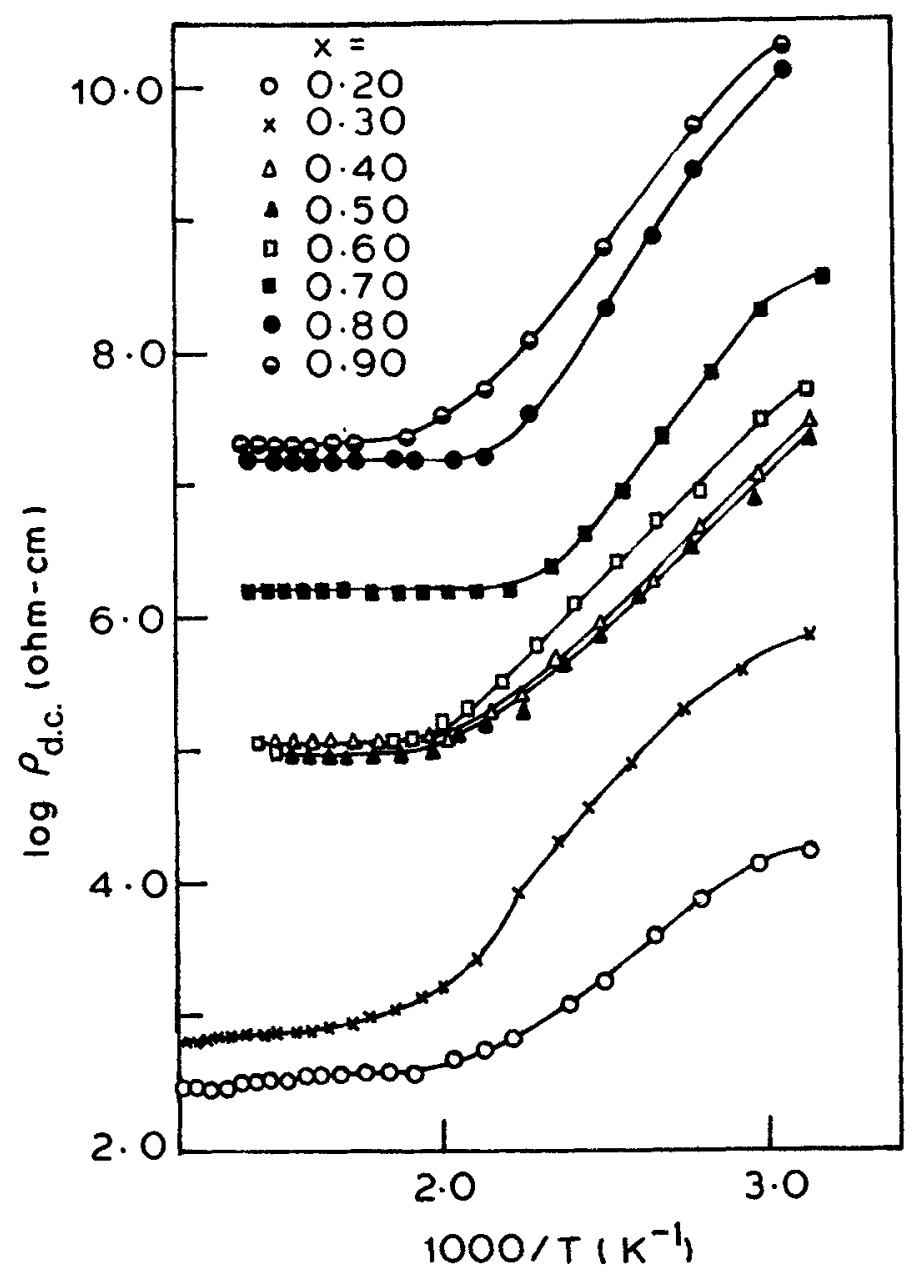

Figure 2. Variation of $\log \rho_{\mathrm{d} . \mathrm{c}} \mathrm{vs} 1000 / T$ for various samples in the system $\mathrm{La}_{1-x} \mathrm{~Pb}_{x-\delta} \mathrm{Co}_{1-x} \mathrm{Ti}_{x} \mathrm{O}_{3-\delta}$. 
where $E_{a}$ is the activation energy for conduction in this region. Values of $E_{a}$ obtained by least square fitting of data are given in table 1 . For samples with $x \leqslant 0 \cdot 3$, a slight decrease in the resistivity is observed with increasing temperature in region 2 , while resistivity is almost independent of temperature for all the other samples in this region. In almost all the samples region 2 starts from $450 \mathrm{~K}$ onwards. The resistivity value increases with $x$. Variations of $\rho_{0 \mathrm{I}}, \rho_{0 \mathrm{II}}$ and $E_{a}$ with $x$ are shown in figure 3.

The a.c. conductivity $\left(\sigma_{\text {a.c. }}\right)$ was measured as a function of temperature and frequency $(f)$. Typical plots of $\log \sigma_{\text {a.c. }}$ vs $\log f$ at a few selected temperatures for samples with $x=0.20,0.30$ and 0.70 are shown in figure 4 . It is observed that all the samples exhibit frequency-independent $\sigma_{\mathrm{acc}}$ for $T \geqslant 473 \mathrm{~K}$. This temperature is greater than $T_{\alpha}$, the temperature of maximum Seebeck coefficient for all the samples. At lower temperatures, frequency dependence of a.c. conductivity was observed above a certain frequency below which it is frequency-independent. This frequency shifts to a higher value with increasing temperature. The variation of $\log \sigma_{\mathrm{acc}}(1 \mathrm{kHz})$ with $1000 / T$ for some of the samples is shown in figure 5 .

DC electrical resistivity behaviour over the entire temperature range and variation of Seebeck coefficient, $\alpha$, with temperature above $T_{\alpha}$ are similar to $\mathrm{LaCoO}_{3}$ for all the samples. The increasing value of both these parameters with $x$ shows that conduction is mainly due to $3 d$ electrons of cobalt ions in this system. Electron transport properties of $\mathrm{LaCoO}_{3}$ have been reported earlier (Bhide et al 1972). It is a p-type semiconductor in which low-spin $\mathrm{Co}^{\mathrm{III}}\left(t_{2 g}^{6} e_{g}^{0}\right)$ ions are separated from highspin $\mathrm{Co}^{3+}\left(t_{2 g}^{4} e_{g}^{2}\right)$ ions by approximately $0.08 \mathrm{eV}$. As $\mathrm{LaCoO}_{3}$ is heated from very low temperature, low-spin $\mathrm{Co}^{\mathrm{III}}$ ions transform to high-spin $\mathrm{Co}^{3+}$ ions upto $200 \mathrm{~K}$. Activation energy of conduction in this region is $0.1 \mathrm{eV}$. Between 200 and $400 \mathrm{~K}$, electron transfer occurs between $\mathrm{Co}^{\mathrm{III}}$ and $\mathrm{Co}^{3+}$. ions generating an intermediate charge transfer states $\mathrm{Co}^{2+}, \mathrm{Co}^{\mathrm{IV}}$ etc. Activation energy of conduction in this region is $\sim 0.2 \mathrm{eV}$ which represents the gap between filled $t_{2 g}^{6}$ orbitals and empty $\sigma^{*}$ band formed from $e_{g}$ orbitals. Between 400 and $650 \mathrm{~K}$, short range ordering of low-spin

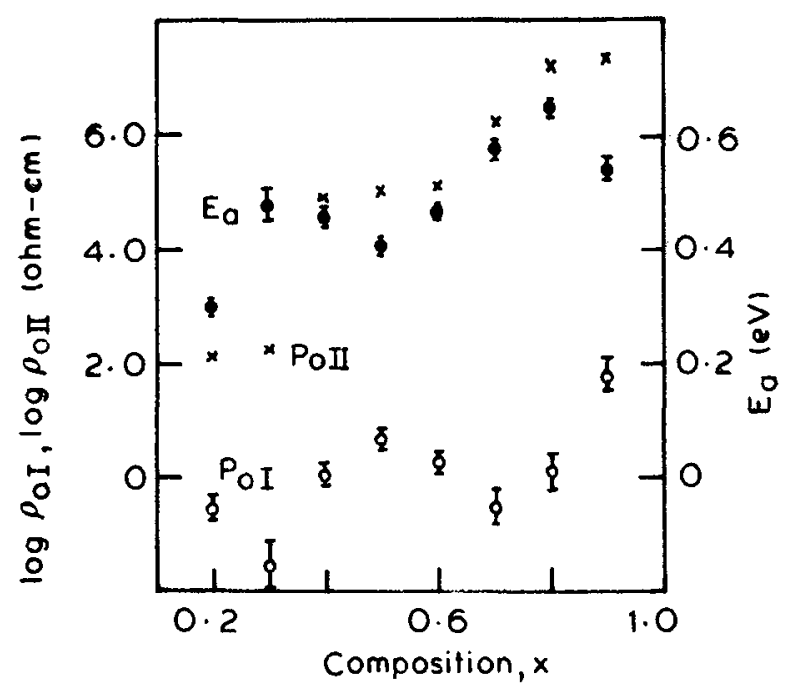

Figure 3. Variation of $\log \rho_{01}(O) \log \rho_{011}(x)$ and $E_{a}(\Theta)$ with composition $x$ in the system $\mathrm{La}_{1-x} \mathrm{~Pb}_{x-\delta} \mathrm{Co}_{1-x} \mathrm{Ti}_{x} \mathrm{O}_{3-\delta}$. 


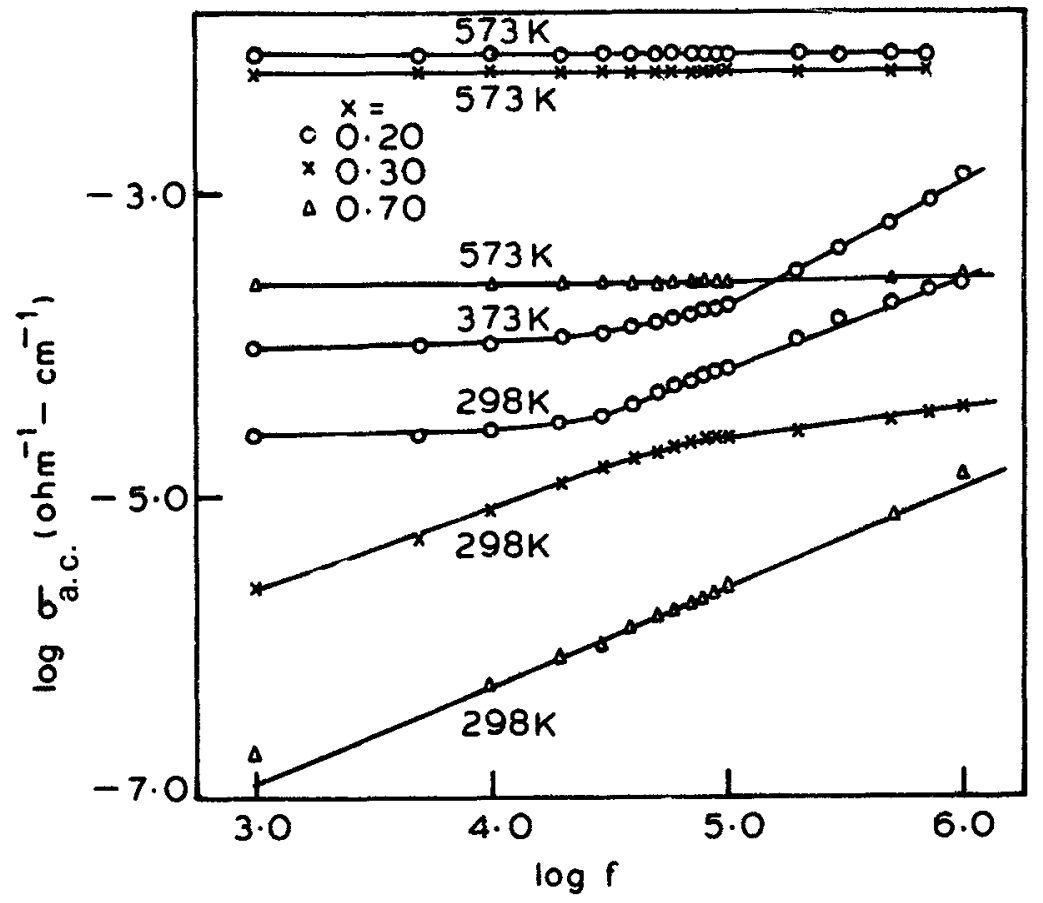

Figure 4. Variation of $\log \sigma_{\mathrm{a} . \mathrm{c}}$ vs $\log f$ for samples with $x=0.20,0.30$ and 0.70 at temperatures $T=298$ and $573 \mathrm{~K}$ in the system $\mathrm{La}_{1-x} \mathrm{~Pb}_{x-\delta} \mathrm{Co}_{1-x} \mathrm{Ti}_{x} \mathrm{O}_{3-\delta}$.

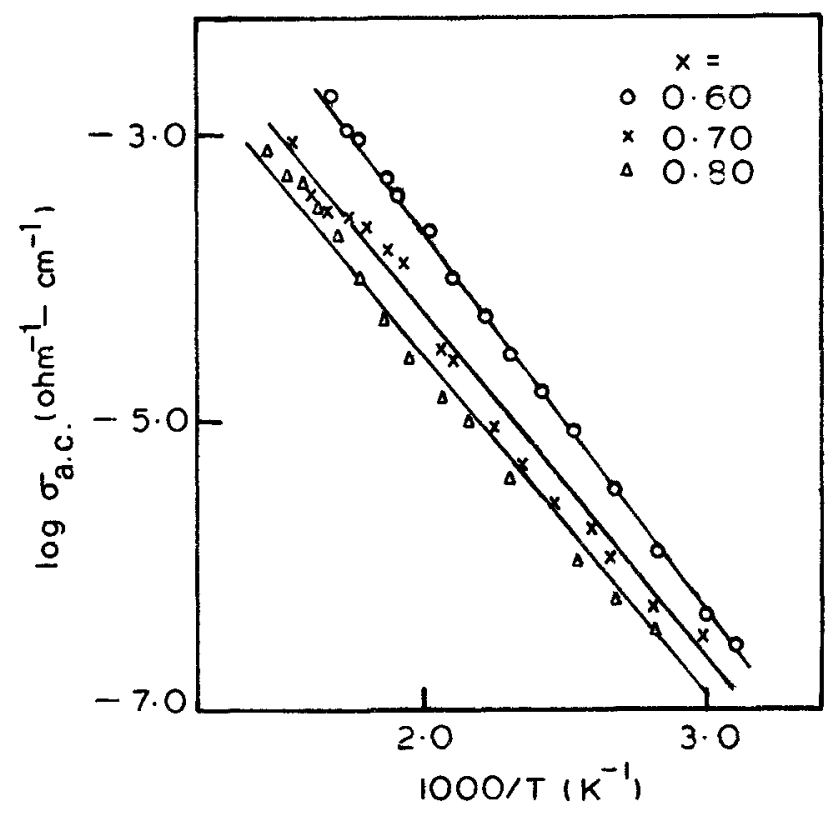

Figure 5. Variation of $\log v_{a c}$ vs $1000 / T$ for samples with $x=0.60,0.70$ and 0.80 in the system $\mathrm{La}_{1-x} \mathrm{~Pb}_{x-\delta} \mathrm{Co}_{1-x} \mathrm{Ti}_{x} \mathrm{O}_{3-\delta}$. 
and high-spin ions occurs leading to a sharp decrease in resistivity with an activation energy of $0.54 \mathrm{eV}$. The sharp decrease in resistivity is ascribed to the small difference in energy $\left(E_{0}\right)$ between low-spin and high-spin ions and to entropy associated with random spin directions (Rao et al 1975). Beyond $650 \mathrm{~K}$ resistivity changes slightly indicating almost saturation of charge carriers and finally it undergoes a first-order transition from localized to collective behaviour of $d$-electrons at $1210 \mathrm{~K}$. Both electrons and holes contribute to conduction in $\mathrm{LaCoO}_{3}$. The electrons are present in orbitals of $e_{g}$ symmetry and holes in orbitals of $t_{2 g}$ symmetry. Their total numbers (trapped plus mobile) are equal. The p-type character of $\mathrm{LaCoO}_{3}$ in the temperature range $300-1200 \mathrm{~K}$ shows mobility of holes is greater than that of electrons. In our system, for $x \leqslant 0.5$, there are some divalent cobalt ions which act as donors. Therefore electron contribution to $\alpha$ increases at low temperatures. This accounts for the small values of $\alpha$ for these values of $x$. The contribution due to electrons decreases sharply upto $T_{\alpha}$ accounting for an overall increase in $\alpha$. Above $T_{\alpha}$, the behaviour is essentially similar to that of $\mathrm{LaCoO}_{3}$. Increase of $\alpha$ below $T_{\alpha}$ may also be due to contribution of hopping of charge carriers among localized sites to the total conductivity. This hopping will be variable in nature due to random distribution of cobalt ions responsible for conduction. In this regime, $\alpha$ would increase with increasing $T$ as observed experimentally (Mott and Davis 1979). Conduction occurs in the extended states above $T_{\alpha}$. This is further supported by our results of a.c conductivity, $\sigma_{\mathrm{a} . \mathrm{c}}$ as a function of frequency at various temperatures (figure 4). AC conductivity is independent of frequency at temperatures greater than $473 \mathrm{~K}$ indicating conduction in the extended states. It is to be noted that $T_{\alpha}$ for all the samples is either less than or equal to $473 \mathrm{~K}$. Below this temperature, the observed frequency dependent of a.c. conductivity in a certain frequency range also shows that there are two contributions to the observed a.c. conductivity: (i) due to conduction in the extended states which is frequency-independent and (ii) frequency dependence contribution due to hopping among localized sites. The second contribution varies according to the relation $\sigma_{\text {a.c. }} \propto \omega^{s}$ where $s$ is a weak function of frequency at a particular temperature. The value of $s$ lies within the range 0.4 to 0.7 for various samples at room temperatures. The value of $s$ decreases with increasing temperature.

The regions of low activation energy of conduction (upto $400 \mathrm{~K}$ ) of $\mathrm{LaCoO}_{3}$ are not observed in all our samples. This may be due to slight changes in the values of crystal field splitting, $\triangle_{c f}$, which occur due to substitution of $\mathrm{Pb}^{2+}$ and $\mathrm{Ti}^{4+}$ in $\mathrm{LaCoO}_{3}$ in various amounts. The generation of $\mathrm{Co}^{3+}$ from $\mathrm{Co}^{\mathrm{II}}$ and subsequent electron transfer from $\mathrm{Co}^{\prime \prime \prime}$ to $\mathrm{Co}^{3+}$ seems to be complete at lower temperatures as compared to $\mathrm{LaCoO}_{3}$ due to such a change. Region I in our samples correspond to $400-650 \mathrm{~K}$ region of electrical conduction in $\mathrm{LaCoO}_{3}$. Activation energies observed in this region are close to that observed in $\mathrm{LaCoO}_{3}$. Above region $\mathrm{I}$, a region indicating saturation of charge carriers similar to that in $\mathrm{LaCoO}_{3}$ (for $T>650 \mathrm{~K}$ ) is observed in all the samples. This shows that conduction process is similar to that in $\mathrm{LaCOO}_{3}$ above $T_{\alpha}$. Only the order of conductivity changes. There are two factors affecting the magnitude of conductivity. One is the decreasing concentration of cobalt ions which decreases conductivity with increasing $x$ as meitioned earlier. The other factor is the change of structure with composition. As is clear from figure $3, E_{a}$ and $\rho_{0 \text { II }}$ change considerably wherever the structure changes.

We would like to compare the behaviour of the present system with those of 
$\mathrm{La}_{1-x} \mathrm{Sr}_{x} \mathrm{CoO}_{3}, \mathrm{LaCo}_{1-x} \mathrm{Ti}_{x} \mathrm{O}_{3}$ and $\mathrm{La}_{1-x} \mathrm{Sr}_{x} \mathrm{Co}_{1-x} \mathrm{Ti}_{x} \mathrm{O}_{3}$. In $\mathrm{La}_{1-x} \mathrm{Sr}_{x} \mathrm{CoO}_{3}$ and $\mathrm{LaCo}_{1-x} \mathrm{Ti}_{x} \mathrm{O}_{3}$, the substitutions of $\mathrm{Sr}^{2+}$ and $\mathrm{Ti}^{3+}$ respectively generate $\mathrm{Co}^{4+}$ ions and $\mathrm{Co}^{2+}$ ions to maintain the overall charge neutrality. Due to the change of valence state of cobalt ions, the properties of these two systems are considerably different from that of $\mathrm{LaCoO}_{3}$ (Bhide et al 1975; Bahadur and Om Parkash 1983; Kumar et al 1986). In $\mathrm{La}_{1-x} \mathrm{Sr}_{x} \mathrm{Co}_{1-x} \mathrm{Ti}_{x} \mathrm{O}_{3}$, due to simultaneous substitution of $\mathrm{Sr}^{2+}$ and $\mathrm{Ti}^{4+}$, though the valence state of cobalt ions remains the same (Kumar et al 1986), the electron transport properties are different from that of $\mathrm{LaCoO}_{3}$. This is due to replacement of $\mathrm{La}^{3+}$ by $\mathrm{Sr}^{2+}$ which increases both the overlap integrals $b^{\sigma}$ and $b^{\pi}$ (Goodenough 1974). In the present system replacement of $\mathrm{La}^{3+}$ by $\mathrm{Pb}^{2+}$ does not affect these parameters much and hence the behaviour of $3 d$-electrons of cobalt ions. Therefore, the behaviour is very similar to that of $\mathrm{LaCoO}_{3}$ except that due to random distribution of cobalt ions, hopping conduction seems to contribute partly to the electron transport in this system. Hopping conduction has also been observed in other systems mentioned above.

\section{Acknowledgements}

The authors express their gratitude to Prof. C N R Rao for useful discussions and constant encouragement. We are thankful to Dr Lakshman Pandey for useful discussions during these investigations. Thanks are due to the Department of Science and Technology, Government of India for financial assistance.

\section{References}

Bahadur D and Om Parkash 1983 J. Solid State Chem. 46197

Bhide V G, Rajoria D S, Rao G R and Rao C N R 1972 Phys. Rev. B6 1021

Bhide V G, Rajoria D S, Rao C N R, Rao G R and Jadhao V G 1975 Phys. Rev. B12 2832

Goodenough J B 1974 Solid state chemistry (ed) C N R Rao (New York: Marcel Dekker) pp 348

Kumar V, Singru R M, Om Parkash and Bahadur D 1985 Phys. Status Solidi B128 223

Mott N F and Davis E A 1979 Electronic processes in non-crystalline materials (Oxford: Clarendon Press) 2nd ed., pp 239

Om Parkash, Prasad C D and Kumar D 1987 J. Solid State Chem. (in press)

Raccah P M and Goodenough J B 1967 Phys. Ret. 155932

Rao C N R, Bhide V G and Mott N F 1975 Philos. Mag. 321277

Wolters M and Burggraaf A J 1974 Phys. Status Solidi A24 341 